This item was submitted to Loughborough's Research Repository by the author.

Items in Figshare are protected by copyright, with all rights reserved, unless otherwise indicated.

\title{
Efficient and fair hybrid TDMA-CSMA for virtualized green wireless networks
}

PLEASE CITE THE PUBLISHED VERSION

http://dx.doi.org/10.1109/VTCFall.2016.7880980

\section{PUBLISHER}

(C) IEEE

VERSION

AM (Accepted Manuscript)

\section{PUBLISHER STATEMENT}

This work is made available according to the conditions of the Creative Commons Attribution-NonCommercialNoDerivatives 4.0 International (CC BY-NC-ND 4.0) licence. Full details of this licence are available at: https://creativecommons.org/licenses/by-nc-nd/4.0/

\section{LICENCE}

CC BY-NC-ND 4.0

\section{REPOSITORY RECORD}

Shoaei, Atoosa Dalili, Mahsa Derakhshani, Saeedeh Parsaeefard, and Tho Le-Ngoc. 2019. "Efficient and Fair Hybrid TDMA-CSMA for Virtualized Green Wireless Networks”. figshare. https://hdl.handle.net/2134/22939. 


\title{
Efficient and Fair Hybrid TDMA-CSMA for Virtualized Green Wireless Networks
}

\author{
Invited Paper
}

\author{
Atoosa Dalili Shoaei*, Mahsa Derakhshani ${ }^{\dagger}$, Saeedeh Parsaeefard*, Tho Le-Ngoc* \\ *Department of Electrical \& Computer Engineering, McGill University, Montreal, QC, Canada \\ $\dagger$ Wolfson School of Mechanical, Electrical and Manufacturing Engineering, Loughborough University, UK \\ Email: atoosa.dalilishoaei@mail.mcgill.ca; m.derakhshani@lboro.ac.uk; saeideh.parsaeifard@mcgill.ca; tho.le-ngoc@mcgill.ca
}

\begin{abstract}
This paper proposes hybrid TDMA-CSMA for virtualized wireless networks, aiming to meet their isolation requirements. In this scheme, high-load users with non-empty queues are proper and potential candidates for TDMA, while others can compete using $p$-persistent CSMA. At each superframe, AP decides on TDMA-CSMA scheduling by taking into account traffic parameters of users and slice reservations to maximize the network utilization, while maintaining slice isolation. The corresponding optimization problem is formulated to dynamically schedule users for TDMA phase and optimally pick $p$ parameter for remaining CSMA users. Using complementary geometric programming (CGP) and monomial approximations, an iterative algorithm is developed to find the optimal solution. The simulation results reveal the performance gains of the proposed algorithm in improving the throughput and keeping isolation in a virtualized wireless network.
\end{abstract}

\section{INTRODUCTION}

The growing demand for data traffic and ubiquitous access necessitates the network infrastructure to be expanded, which immediately results in larger energy requirement in wireless networks. Such increase in energy consumption causes rising greenhouse gas emission, which has been a major environmental concern. Thus, it is urgent and crucial to develop green wireless technologies, which can compensate for the high demand for wireless traffic and energy consumption by more sustainable solutions for capacity growth [1]. One promising technology to improve energy efficiency and enable green communication is wireless virtualization. In virtualized wireless networks (VWN), a single network infrastructure can be shared among different service providers (SPs). Thus, by using renewable energy resources for the network infrastructure in VWN, a significant amount of energy can be saved, and, at the same time, SPs can reduce their costs.

One of the key requirement in VWN is to support isolation among different SPs. In this context, isolation means that any change in one SP such as number of users, mobility of users, and fluctuation of channel status, should not cause any change in resource allocation for other SPs [2]. Moreover, since wireless resources are scarce, high utilization is also expected. The problem is that these two requirements of VWN, i.e., efficient resource utilization and isolation, are conflicting specifically in uplink transmission as traffic is generated at users, while resources are allocated by the access point (AP).
For instance, in order to provide strict isolation among slices, a time division multiple access (TDMA)-based scheme can be applied, in which an exclusive timeshare is reserved for each SP based on its requirement. However, such allocation could lead to underutilization for an unsaturated network, where users may not have any packet to transmit in their assigned time-slots. On the other hand, random access protocols such as carrier sense multiple access (CSMA) have more dynamic and opportunistic nature [3] and can overcome the utilization inefficiency. But in such protocols, the total time used by each SP should be defined as time spent for both successful and unsuccessful transmissions. Therefore, each SP would affect the performance of other SPs by increasing the collision time and thus isolation cannot be provided [4].

In this paper, we propose and adaptively optimize a channel access scheme that uses a hybrid TDMA-CSMA to cope with the aforementioned issues. In the context of conventional wireless networks, the combinational use of TDMA and CSMA has been studied [3], [5], [6]. However, none of these works consider virtualized networks in which meeting SP requirements adheres challenges to network utilization maximization. In order to address this issue, we propose a hybrid MAC in which $p$-persistent CSMA is applied in CSMA phase. In this approach, assuming that AP is aware of packet arrival probabilities of users, at each superframe, it decides whether each user should transmit in TDMA or CSMA phase. Moreover, for CSMA users, corresponding $p$ is also determined. This decision making problem is formulated by an optimization problem, in which the goal is to maximize the overall throughput of network at each superframe subject to the SP reservations. In order to solve the formulated non-convex problem, we propose an efficient and tractable iterative approach in which at each iteration, via applying different types of transformation techniques and using arithmetic geometric mean inequality, the formulated problem can be transformed into the geometric programming (GP).

The rest of this paper is organized as follows. We first introduce our system model in Section II. Section III presents the problem formulation. Subsequently, an iterative CGP-based hybrid TDMA-CSMA scheduling is presented in Section IV. Section V presents the simulation results. Finally, we provide some concluding remarks in Section VI. 


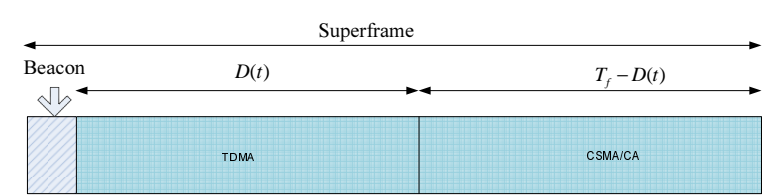

Fig. 1: Superframe structure of the proposed MAC for a virtualized 802.11 WLAN

\section{System MODEL}

\section{A. Network Model and Frame Structure}

We consider an IEEE 802.11-based WLAN with a single AP. The AP serves the users of $\mathcal{K}=\{1, \cdots, K\}$ different SPs (also referred to as slices). In this setup, each slice $k$ has the set of its own subscribed users denoted by $\mathcal{N}_{k}$, where $\left|\mathcal{N}_{k}\right|=N_{k}$ is the number of users at slice $k$. Thus, the total number of all users over all slices is $N_{\mathrm{u}}=\sum_{k=1}^{K} N_{k}$.

Time is divided into superframes with length of $T_{f}$, indexed by $t$. As shown in Figure 1, each superframe begins with a beacon issued by AP, which consists TDMA scheduling information and $p$ values of $p$-persistent CSMA. The beacon is followed by the TDMA phase with duration of $D(t)\left(\leq D_{\max }\right)$ for scheduled users, and the CSMA phase of length $T_{f}-D(t)$. During the CSMA phase, $p$-persistent CSMA protocol runs, where each time-slot with duration of $T_{s}$ is divided into backoff units. Regarding the required QoS per slice, it is assumed that each SP $k$ can reserve time for $r_{k}$ per superframe.

We assume each user $n_{k}$ has queue with maximum length of $Q_{\text {max }}$, and at each superframe, with probability of $a_{n_{k}}$, a new packet is added to the queue if its length is below than $Q_{\max }$. Otherwise, the packet is discarded. Furthermore, we assume that AP is aware of packet arrival probabilities of users and it keeps a vector denoted by $\boldsymbol{V}(t)$, where $v_{n_{k}}$ denotes the last time that AP has received packet from user $n_{k}$. Moreover, each time the user sends a packet, it piggybacks an extra bit (denoted by $\left.q_{n_{k}}(t)\right)$ telling if its queue is empty $\left(q_{n_{k}}(t)=0\right.$ ) or non-empty $\left(q_{n_{k}}(t)=1\right.$, i.e., it has packets backlogged in the queue to transmit). Therefore, at each superframe $t$, the AP updates the probability that user $n_{k}$ has non-empty queue at $t$ as

$$
\theta_{n_{k}}(t)= \begin{cases}1-\left(1-a_{n_{k}}\right)^{t-v_{n_{k}}(t)}, & \text { if } q_{n_{k}}\left(v_{n_{k}}(t)\right)=0 \\ 1 & \text { if } q_{n_{k}}\left(v_{n_{k}}(t)\right)=1\end{cases}
$$

\section{B. An Analytical Model for p-persistent CSMA}

In the CSMA phase, a user with a non-empty packet performs the channel sensing. If the channel is detected busy, the user $n_{k}$ with a non-empty queue waits until channel becomes idle, and then transmits the packet with probability $p_{n_{k}}$. In the following, we model the throughput of $p$-persistent CSMA protocol in an unsaturated mode. Let $P_{\text {idle }}$ be the probability that channel is idle in a backoff unit. This probability is calculated as

$$
P_{\text {idle }}=\prod_{k \in \mathcal{K}} \prod_{n_{k} \in \mathcal{N}_{k}}\left(1-\theta_{n_{k}} p_{n_{k}}\right)
$$

where $\theta_{n_{k}} p_{n_{k}}$ represents the transmission probability of user $n_{k}$. A transmitted packet will be received successfully if exactly one user transmits on the channel. Thus, the probability of successful transmission initiated by user $n_{k}$ can be obtained as

$$
P_{\text {succ }}^{n_{k}}=\theta_{n_{k}} p_{n_{k}} \prod_{k \in \mathcal{K}} \prod_{n_{k}^{\prime} \in \mathcal{N}_{k}, n_{k}^{\prime} \neq n_{k}}\left(1-\theta_{n_{k}^{\prime}} p_{n_{k}^{\prime}}\right) .
$$

As introduced in [7], we define the normalized throughput of user $n_{k}$ (denoted by $\rho_{n_{k}}$ ) as the fraction of time the channel is used for its successful transmission. Accordingly, $\rho_{n_{k}}$ can be written as

$$
\rho_{n_{k}}=\frac{P_{\text {succ }}^{n_{k}} T_{s}}{P_{\text {idle }} \delta+\left(1-P_{\text {idle }}\right) T_{s}},
$$

where $\delta$ is the duration of a backoff unit and $T_{s}$ is the duration of a successful transmission, which includes the data transmission for a fixed time, inter-frame spaces, and signaling overheads. Since signaling and inter-frame spaces are relatively small compared with data transmission length, we approximately assume that both collision and successful transmissions are of the same size (i.e., $T_{s}$ ). Consequently, the denominator in (4) represents the expected length of a general time-slot.

By introducing a new variable, i.e., $y_{n_{k}}=\frac{\theta_{n_{k}} p_{n_{k}}}{1-\theta_{n_{k}} p_{n_{k}}}$, we can simplify (4). To this end, first, we rewrite $P_{\text {idle }}$ and $P_{\text {succ }}^{n_{k}}$ in terms of $y_{n_{k}}$ as

$$
\begin{aligned}
P_{\text {idle }} & =\frac{1}{\prod_{k \in \mathcal{K}} \prod_{n_{k} \in \mathcal{N}_{k}}\left(1+y_{n_{k}}\right)} \\
P_{\text {succ }}^{n_{k}} & =\frac{y_{n_{k}}}{\prod_{k \in \mathcal{K}} \prod_{n_{k} \in \mathcal{N}_{k}}\left(1+y_{n_{k}}\right)}=y_{n_{k}} P_{\text {idle }} .
\end{aligned}
$$

Then, we obtain $\rho_{n_{k}}$ in terms of $y_{n_{k}}$ as

$$
\rho_{n_{k}}=\frac{y_{n_{k}}}{\prod_{k \in \mathcal{K}} \prod_{n_{k} \in \mathcal{N}_{k}}\left(1+y_{n_{k}}\right)-t^{\prime}},
$$

where $t^{\prime}=\frac{T_{s}-\delta}{T_{s}}$. In addition to the throughput of each user, another performance metric, total access airtime, is important to measure and preserve isolation in the context of virtualized wireless networks. The total CSMA access airtime of user $n_{k}$, in which both collisions and successful transmissions are included, can be defined as

$$
\tau_{\mathrm{cs}}^{n_{k}}=\frac{\left(1-P_{\mathrm{idle}}^{n_{k}}\right) T_{s}}{P_{\text {idle }} \delta+\left(1-P_{\text {idle }}\right) T_{s}} .
$$

This metric can also be represented in terms of $y_{n_{k}}$ as

$$
\tau_{\mathrm{cs}}^{n_{k}}=\frac{y_{n_{k}} \prod_{k \in \mathcal{K}} \prod_{n_{k}^{\prime} \in \mathcal{N}_{k}, n_{k}^{\prime} \neq n_{k}}\left(1+y_{n_{k}^{\prime}}\right)}{\prod_{k \in \mathcal{K}} \prod_{n_{k} \in \mathcal{N}_{k}}\left(1+y_{n_{k}}\right)-t^{\prime}} .
$$

\section{ThroughPUT-MAXIMIZATION HYBRID}

\section{TDMA-CSMA FOR VWN: PROBLEM FORMULATION}

In order to benefit from TDMA isolation power and CSMA opportunistic nature we propose hybrid TDMA-CSMA. In this scheme, the scheduling algorithm determines which users should transmit in the TDMA phase, based on traffic demand of each user and required reservation of each slice. Users that 
are not scheduled for TDMA transmission have a chance to transmit in the remaining time of superframe using $p$-persistent CSMA protocol, where parameter $p$ of each individual user is determined by the proposed algorithm.

Here, we present the formulation for throughput maximization of this hybrid MAC protocol, assuming that statistical traffic parameters of users (i.e., $a_{n_{k}}$ ) are known by AP. We denote by $\boldsymbol{X}(t)=\left[x_{n_{k}}(t)\right]_{\forall n_{k}}$ the vector representing the timeslot allocation of all users at superframe $t$. More specifically, $x_{n_{k}}(t)$ is a binary variable indicating whether a time-slot is allocated to user $n_{k}$ in superframe $t$ (i.e., $x_{n_{k}}(t)=1$ ) or not (i.e., $x_{n_{k}}(t)=0$ ).

The expected throughput, which is defined as the number of successfully transmitted packets in superframe $t$, considered as the sum of the TDMA- and CSMA-phase throughputs. The throughput associated with the TDMA phase can be written as

$$
S_{\mathrm{td}}(t)=\sum_{k \in \mathcal{K}} \sum_{n_{k} \in \mathcal{N}_{k}} \theta_{n_{k}}(t) x_{n_{k}}(t) .
$$

Moreover, the CSMA-phase throughput can be computed as

$$
S_{\mathrm{cs}}(t)=T_{\mathrm{cs}}(t) \sum_{k \in \mathcal{K}} \sum_{n_{k} \in \mathcal{N}_{k}} \rho_{n_{k}},
$$

where $T_{c s}(t)$ denotes the duration of CSMA phase in superframe $t$. Taking into account the number of scheduled users for TDMA phase, $T_{\mathrm{cs}}(t)$ can be represented as

$$
T_{\mathrm{cs}}(t)=T_{f}-T_{s} \sum_{k \in \mathcal{K}} \sum_{n_{k} \in \mathcal{N}_{k}} x_{n_{k}}(t) .
$$

Further to throughput for TDMA and CSMA phases, we need to compute the total airtime access of each slice in order to provide isolation among slices. The instantaneous expected total airtime access for slice $k$ can be obtained as

$$
\tau_{k}(t)=\sum_{n_{k} \in \mathcal{N}_{k}}\left[T_{s} x_{n_{k}}(t)+T_{\mathrm{cs}}(t) \tau_{\mathrm{cs}}^{n_{k}}(t)\right] .
$$

In (13), the first term represents the time assigned to users belonging to slice $k$ during the TDMA phase and the second term indicates the average total access time of users of slice $k$ in CSMA phase.

Finally, at each superframe $t$, the AP should solve the following optimization problem in order to obtain $X$ and $Y$.

$$
\begin{aligned}
& \max _{\boldsymbol{X}, \boldsymbol{Y}} S_{\mathrm{td}}(t)+S_{\mathrm{cs}}(t), \quad \text { subject to, } \\
& \text { C1: } \tau_{k}(t) \geq r_{k}, \quad \forall k \in \mathcal{K} \\
& \text { C2: } x_{n_{k}} p_{n_{k}}=0, \quad \forall k \in \mathcal{K}, \forall n_{k} \in \mathcal{N}_{k} \\
& \text { C3: } T_{s} \sum_{k \in \mathcal{K}} \sum_{n_{k} \in \mathcal{N}_{k}} x_{n_{k}} \leq D_{\max } .
\end{aligned}
$$

In this optimization problem, the objective function represents the total throughput of network in both TDMA and CSMA phases for superframe $t$. The first constraint is to guarantee that reservation of each slice is met. Moreover, the second one ensures that user $n_{k}$ is only selected for TDMA or CSMA, not both. Finally, the last constraint is to limit the number of users that transmit in TDMA phase. In the rest of paper, $t$ is omitted in all equations for the sake of simplicity.
Substituting (10), (11), (12), and (13), the optimization problem in (14) can be written as

$$
\max _{\boldsymbol{X}, \boldsymbol{Y}} \sum_{k \in \mathcal{K}} \sum_{n_{k} \in \mathcal{N}_{k}}\left[\theta_{n_{k}} x_{n_{k}}+\frac{y_{n_{k}}\left(T_{f}-T_{s} \sum_{k \in \mathcal{K}} \sum_{n_{k} \in \mathcal{N}_{k}} x_{n_{k}}\right)}{\prod_{k \in \mathcal{K}} \prod_{n_{k} \in \mathcal{N}_{k}}\left(1+y_{n_{k}}\right)-t^{\prime}}\right]
$$

subject to:

$\mathrm{C} 1: \frac{y_{n_{k}} \prod_{k \in \mathcal{K}} \prod_{n_{k}^{\prime} \in \mathcal{N}_{k}, \neq n_{k}}\left(1+y_{n_{k}^{\prime}}\right)}{\prod_{k \in \mathcal{K}} \prod_{n_{k} \in \mathcal{N}_{k}}\left(1+y_{n_{k}}\right)-t^{\prime}} \times$

$$
\left(T_{f}-T_{s} \sum_{k \in \mathcal{K}} \sum_{n_{k} \in \mathcal{N}_{k}} x_{n_{k}}\right) \geq r_{k}, \forall k \in \mathcal{K}
$$

C2: $x_{n_{k}} p_{n_{k}}=0, \quad \forall k \in \mathcal{K}, \forall n_{k} \in \mathcal{N}_{k}$

C3: $T_{s} \sum_{k \in \mathcal{K}} \sum_{n_{k} \in \mathcal{N}_{k}} x_{n_{k}} \leq D_{\max }$.

It is clear that the optimization problem in (15) has a nonconvex objective function due to couplings in the CSMA throughput and involves non-linear constraints with the combination of continuous and binary variables, i.e., $y_{n_{k}}\left(y_{n_{k}} \geq 0\right)$ and $x_{n_{k}}\left(x_{n_{k}} \in\{0,1\}\right)$. Consequently, (14) is a non-convex mixed-integer, NP-hard optimization problem. Therefore, an efficient algorithm with reasonable computational complexity is needed to solve this scheduling problem.

\section{HYBRID TDMA-CSMA SCHEDULING VIA CGP}

To solve the hybrid TDMA-CSMA scheduling problem in (15), we propose an iterative algorithm by applying complementary geometric programming, as discussed in the following sub-sections.

\section{A. A brief review of complementary geometric programming}

A geometric programming (GP) is an optimization problem of the form

$$
\begin{array}{cl}
\min _{\boldsymbol{x}} & f_{0}(\boldsymbol{x}) \\
\text { s.t. : } & f_{i}(\boldsymbol{x}) \leq 1, i=1,2, \ldots, I \\
& g_{j}(\boldsymbol{x})=1, j=1,2, \ldots, J,
\end{array}
$$

where $\boldsymbol{x}=\left[x_{1}, \ldots, x_{N}\right]$ is a non-negative vector of optimization variables, $g_{j}(\boldsymbol{x})=c_{i} \prod_{n=1}^{N} x_{n}^{b_{i, n}}$ for all $j$ are monomial functions, and $f_{i}(\boldsymbol{x})=\sum_{k=1}^{K_{j}} c_{j, k} \prod_{n=1}^{N} x_{n}^{b_{j, k, n}}$ are posynomial functions for $i=0, \ldots, I$, where the multiplicative constants are positive (i.e., $c_{i}, c_{j, k}>0$ ) and $b_{i, n}, b_{j, k, n} \in \boldsymbol{R}$.

There is a class of non-convex optimization problems called complementary geometric programming (CGP), which potentially looks like an extension of GP. In particular, a complementary geometric programming (CGP) can be presented as

$$
\begin{array}{cl}
\min _{\boldsymbol{x}} & P_{0}(\boldsymbol{x}) \\
\text { s.t. : } & P_{i}(\boldsymbol{x}) \leq 1, i=1, \ldots, I, \\
& Q_{j}(\boldsymbol{x})=1, j=1, \ldots, J,
\end{array}
$$

where $P_{0}(\boldsymbol{x})$ is a posynomial and $P_{i}(\boldsymbol{x})=\frac{p_{i}(\boldsymbol{x})}{p_{i}^{+}(\boldsymbol{x})}$ for all $i=1, \ldots, I$, in which $p_{i}(\boldsymbol{x})$ and $p_{i}^{+}(\boldsymbol{x})$ are posynomial 
functions. Moreover, $Q_{j}(\boldsymbol{x})=\frac{q_{j}(\boldsymbol{x})}{q_{j}^{+}(\boldsymbol{x})}$ for all $j$, in which $q_{j}(\boldsymbol{x})$ are monomial and $q_{j}^{+}(\boldsymbol{x})$ are posynomial functions.

In the following, we describe monomial approximation which is useful to transform the problem into GP. Let $g_{i}(\boldsymbol{x})=\sum_{k=1}^{K_{i}} m_{i, k}^{g}(\boldsymbol{x})$, where $m_{i, k}^{g}$ is a monomial. Using the arithmetic-geometric mean inequality, at iteration $l, g_{i}(\boldsymbol{x})$ can be approximated as

$$
\widetilde{g}_{i}(\boldsymbol{x}(l))=\prod_{k=1}^{K_{i}}\left(\frac{m_{i, k}^{g}(\boldsymbol{x}(l))}{\alpha_{i, k}(\boldsymbol{x}(l))}\right)^{\alpha_{i, k}(\boldsymbol{x}(l))},
$$

The parameters $\alpha_{i, k}(\boldsymbol{x}(l))$ and $\zeta_{j, k}(\boldsymbol{x}(l))$ can be computed as

$$
\alpha_{i, k}(\boldsymbol{x}(l))=\frac{m_{i, k}^{g}((\boldsymbol{x}(l-1))}{g_{i}((\boldsymbol{x}(l-1))}, \quad \forall i, k
$$

where $x(l-1)$ is the value of last-round solution of the optimization problem. It is proved that arithmetic-geometric mean approximation gives the best local monomial approximation for a posynomial function [8].

Using the approximations in (18), in each iteration, the optimization problem in (17) would be in the form of a standard GP problem. Consequently, the optimal solution can be achieved by iteratively applying monomial approximations and solving a series of GPs [9], [10].

\section{B. Hybrid TDMA-CSMA Scheduling via CGP}

The formulated problem in (15) is non-convex and thus intractable to solve. However, it potentially looks like a CGP problem. In this section, we describe how to transform the problem into a CGP form and then solve it iteratively by applying monomial approximations as discussed in subsection IV-A. First, we can maximize the objective function, by minimizing its negative. However, in GP the objective function should be positive, and this can be done by adding a sufficiently large constant $M$. Moreover, we introduce two auxiliary variables $d=\prod_{k \in \mathcal{K}} \prod_{n_{k} \in \mathcal{N}_{k}}\left(1+y_{n_{k}}\right)-t^{\prime}$ and $T_{\mathrm{cs}}=T_{f}-T_{s} \sum_{k \in \mathcal{K}} \sum_{n_{k} \in \mathcal{N}_{k}} x_{n_{k}}$.

By replacing these auxiliary variables with their corresponding terms and applying the aforementioned changes in objective function, the optimization problem becomes

$$
\min _{\boldsymbol{X}, \boldsymbol{Y}, T_{\mathrm{cs}}, d} M-\sum_{k \in \mathcal{K}} \sum_{n_{k} \in \mathcal{N}_{k}}\left[\theta_{n_{k}} x_{n_{k}}+T_{\mathrm{cs}} y_{n_{k}} d^{-1}\right]
$$

subject to:

$$
\begin{aligned}
& \mathrm{C} 1: \sum_{n_{k} \in \mathcal{N}_{k}}\left[T_{s} x_{n_{k}}+T_{\mathrm{cs}} y_{n_{k}} d^{-1} \prod_{\forall k} \prod_{n_{k}^{\prime} \in \mathcal{N}_{k}, \neq n_{k}}\left(1+y_{n_{k}^{\prime}}\right)\right] \geq r_{k} \text {, } \\
& \forall k \in \mathcal{K}
\end{aligned}
$$

$\mathrm{C} 2: x_{n_{k}} p_{n_{k}}=0, \forall k \in \mathcal{K}, \forall n_{k} \in \mathcal{N}_{k}$,

C3: $T_{s} \sum_{k \in \mathcal{K}} \sum_{n_{k} \in \mathcal{N}_{k}} x_{n_{k}} \leq D_{\max }$,

C4: $d=\prod_{k \in \mathcal{K}} \prod_{n_{k} \in \mathcal{N}_{k}}\left(1+y_{n_{k}}\right)-t^{\prime}$,

C5: $T_{\mathrm{cs}}=T_{f}-T_{s} \sum_{k \in \mathcal{K}} \sum_{n_{k} \in \mathcal{N}_{k}} x_{n_{k}}$.

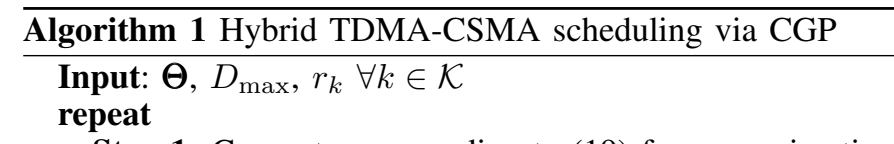

Step 1: Compute $\alpha$ according to (19) for approximation of denominators $\mathrm{C}\{1,2,4-6\}$ at $\left(X, Y, T_{\mathrm{CS}}, d, x_{0}\right)$

Step 2: $\left(\boldsymbol{X}^{\prime}, \boldsymbol{Y}^{\prime}, T_{\mathrm{CS}}^{\prime}, d^{\prime}, x_{0}^{\prime}\right) \leftarrow\left(\boldsymbol{X}, \boldsymbol{Y}, T_{\mathrm{CS}}, d, x_{0}\right)$

Step 3: $\left(\boldsymbol{X}, \boldsymbol{Y}, T_{\mathrm{CS}}, d, x_{0}\right) \leftarrow \mathrm{CVX}$-solver (GP problem in (21)); use $\alpha$ values from Step 1 to approximate the monomial terms in denominators

until $\left|x_{0}-x_{0}^{\prime}\right|<\eta$

$\forall n_{k}: X\left(n_{k}\right)=1$ if $X\left(n_{k}\right)>\bar{X}$, otherwise $X\left(n_{k}\right)=0$ $\forall n_{k}: p_{n_{k}}=y_{n_{k}} /\left(1+y_{n_{k}}\right) \theta_{n_{k}}$

Output: $X, P$

In (20), the objective function is not posynomial because of the negative multiplicative in the second term. This can be handled by introducing and minimizing a new auxiliary variable $x_{0}$ in addition to guaranteeing the constraint C6 in (21). Finally, we reach to the following optimization problem

$$
\begin{aligned}
& \min _{\boldsymbol{X}, \boldsymbol{Y}, T_{\mathrm{cs}}, d, x_{0}} x_{0}, \text { subject to: } \\
& \mathrm{C} 1: \frac{r_{k}}{\sum_{n_{k} \in \mathcal{N}_{k}}\left[T_{s} x_{n_{k}}+T_{\mathrm{cs}} y_{n_{k}} d^{-1} \prod_{\forall k} \prod_{n_{k}^{\prime} \in \mathcal{N}_{k}, \neq n_{k}}\left(1+y_{n_{k}^{\prime}}\right)\right]} \leq 1 \text {, } \\
& \forall k \in \mathcal{K} \\
& \mathrm{C} 2: \frac{1}{1+x_{n_{k}} p_{n_{k}}}=1 \quad, \forall k \in \mathcal{K}, n_{k} \in \mathcal{N}_{k} \text {, } \\
& \text { C3: } T_{s} D_{\max }^{-1} \sum_{k \in \mathcal{K}} \sum_{n_{k} \in \mathcal{N}_{k}} x_{n_{k}} \leq 1 \text {, } \\
& \text { C4: } \frac{\prod_{k \in \mathcal{K}} \prod_{n_{k} \in \mathcal{N}_{k}}\left(1+y_{n_{k}}\right)}{t^{\prime}+d}=1 \text {, } \\
& \text { C5: } \frac{T_{f}}{T_{\mathrm{cs}}+T_{s} \sum_{k \in \mathcal{K}} \sum_{n_{k} \in \mathcal{N}_{k}} x_{n_{k}}}=1 \quad, \forall k \in \mathcal{K}, n_{k} \in \mathcal{N}_{k} \text {, } \\
& \text { C6: } \frac{M}{x_{0}+\sum_{k \in \mathcal{K}} \sum_{n_{k} \in \mathcal{N}_{k}}\left[\theta_{n_{k}} x_{n_{k}}+T_{\mathrm{cs}} y_{n_{k}} d^{-1}\right]} \leq 1 \text {. }
\end{aligned}
$$

In this optimization problem, all upper-bound inequality constraints are in the form of ratio between two posynomials and equality constraints are in the form of ratio between a monomial and a posynomial, as in a CGP problem. As discussed in sub-section IV-A, the algorithm for treating CGP consists of monomial approximations and solving a sequence of resulting GP problems until convergence happens. The algorithm to solve (21) is described in Algorithm 1.

\section{ILlustrative ResUlts}

In this section, we present the simulation results to evaluate proposed hybrid TDMA-CSMA scheme. The simulation is done in MATLAB and GP problems are solved using CVX. For performance evaluation, we study system throughput, which is defined as number of packets successfully transmitted in a superframe $(\mathrm{pckt} / \mathrm{SF})$. The results are compared to $p$ persistent CSMA where parameter $p$ is set to 0.05 for all users.

We consider a network with two slices. We assume that each superframe consists of 19 time-slots and length of each 


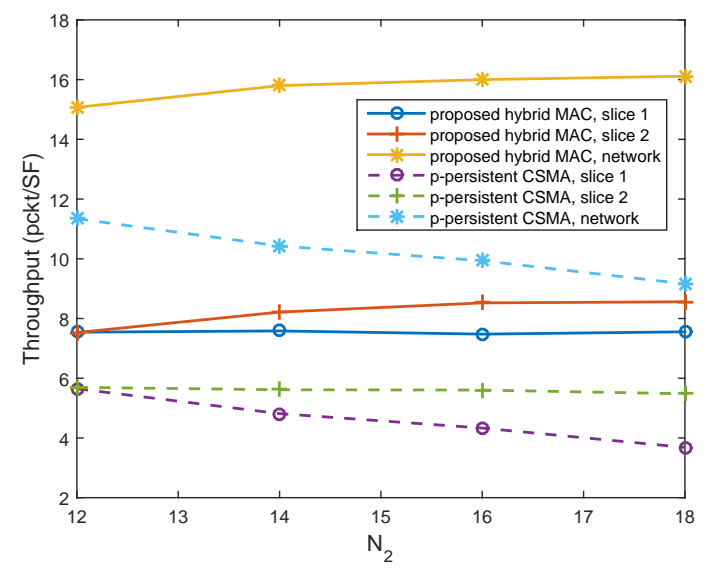

Fig. 2: Throughput versus $N_{2}$ (homogeneous slices)

time-slot, $T_{\mathrm{s}}$, is equal to 12 backoff units. The simulation time is set to 100 superframes and each simulation is repeated 10 times. We also set reservation of each slice equal to $r_{k}=6$ and maximum length of queue equal to $Q_{\max }=4$.

First, we assume a homogeneous scenario, where both slices have the same packet arrival probabilities. To study how well the isolation among slices can be protected in the presence of a variation in one slice, the throughput performance of both slices is plotted for different numbers of users in slice 2, while no parameter has changed in slice 1 . In particular, the packet arrival probabilities of slices are set as $\boldsymbol{A}_{1}=\left\{[0.9]_{4},[0.5]_{8}\right\}$ and $\boldsymbol{A}_{2}=\left\{[0.9]_{4},[0.5]_{N_{2}-4}\right\}$. As shown in Figure 2, by increasing $N_{2}$, throughput of slice 1 degrades slightly while its reservation is still met. However, the throughput of slice 2 increases since more packets are generated in this slice and therefore assigned time-slots to this slice are left idle with lower probability. On the other hand, for using only p-persistent CSMA throughput of slice 1 degrades as $N_{2}$ increases since the users get less chance to transmit their packets. Moreover, the network throughput decreases as well due to larger number of collisions happen.

Furthermore, we investigate the throughput results for a scenario of heterogeneous slices, i.e., with different traffic parameters. In this scenario, we set $\boldsymbol{A}_{1}=\left\{[0.6]_{4},[0.5]_{4},[0.4]_{4},[0.3]_{4},[0.2]_{4}\right\} \quad$ and $\boldsymbol{A}_{2}=\left\{[0.95]_{2},[0.9]_{2},[0.85]_{2},[0.8]_{2},[0.5]_{N_{2}-8}\right\}$, in which $N_{1}=20$ and $N_{2} \geq 10$. More specifically, in this scenario, most users from slice 2 have higher packet arrival probabilities and, hence, most TDMA time-slots are assigned to these users. As shown in Figure 3, by increasing $N_{2}$, throughput of slice 1 remains unchanged while for using only $p$-persistent CSMA the throughput decreases, which shows the effectiveness of the proposed algorithm in providing slice isolation.

\section{CONCLUSION}

This paper presents a hybrid TDMA-CSMA scheduling, where TDMA and CSMA are used for users with high and low packet transmission probabilities, respectively. This

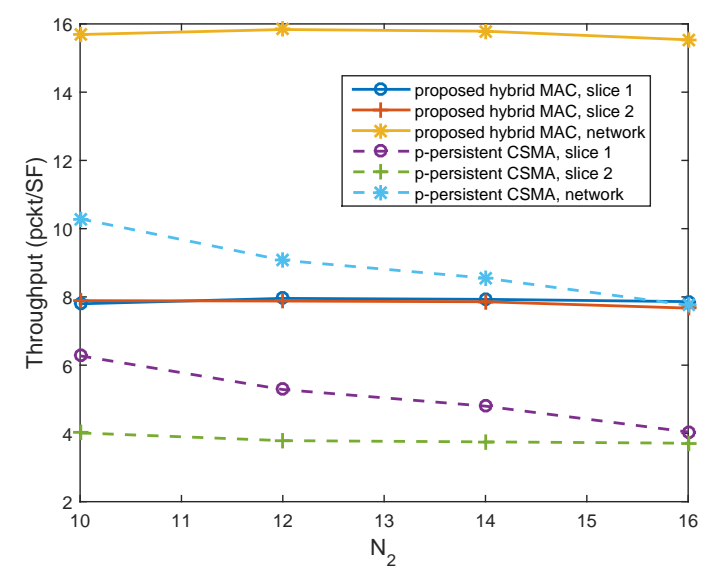

Fig. 3: Throughput versus $N_{2}$ (heterogeneous slices)

scheduling is formulated as an optimization problem with the objective to maximize the network throughput subject to constraints on slice reservations. In order to solve this problem, we show that this problem belongs to CGP, which can be efficiently solved by applying monomial approximations and solving the sequence of resulting GP problems. The output of this optimization problem determines TDMA users and $p$ parameter of CSMA users. Finally, using simulation results, we show the effectiveness of the proposed algorithm to ensure reservation of different slices despite their user distribution and traffic properties.

\section{REFERENCES}

[1] Y. Chen, S. Zhang, S. Xu, and G. Y. Li, "Fundamental trade-offs on green wireless networks," IEEE Commun. Magn.,, vol. 49, no. 6, pp. 30-37, Jun. 2011.

[2] R. Kokku et al., "NVS: a virtualization substrate for WiMAX networks," in Proc. ACM conf. Mobile comput. netw., 2010.

[3] B. Shrestha, E. Hossain, and K. W. Choi, "Distributed and centralized hybrid CSMA/CA-TDMA schemes for single-hop wireless networks," IEEE Trans. Wireless Commun., vol. 13, no. 7, pp. 4050-4065, Jul. 2014.

[4] A. Checco and D. J. Leith, "Fair virtualization of 802.11 networks," Networking, IEEE/ACM Transactions on, vol. 23, no. 1, pp. 148-160, 2015.

[5] B. Shrestha, K. W. Choi, and E. Hossain, "A dynamic time slot allocation scheme for hybrid CSMA/TDMA MAC protocol," IEEE Wireless Commun. Let., vol. 2, no. 5, pp. 535-538, Oct. 2013.

[6] B. Shrestha, E. Hossain, S. Camorlinga, R. Krishnamoorthy, and D. Niyato, "An optimization-based GTS allocation scheme for IEEE 802.15. 4 MAC with application to wireless body-area sensor networks," in Proc. IEEE Intl. Conf. Commun. (ICC), 2010, pp. 1-6.

[7] G. Bianchi, "Performance analysis of the IEEE 802.11 distributed coordination function," IEEE J. Sel. Areas Commun., vol. 18, no. 3, pp. 535-547, Mar. 2000.

[8] S. Boyd and L. Vandenberghe, Convex optimization. Cambridge university press, 2004.

[9] G. Xu, "Global optimization of signomial geometric programming problems," European Journal of Operational Research, vol. 233, no. 3, pp. 500-510, 2014.

[10] M. Derakhshani, X. Wang, T. Le-Ngoc, and A. Leon-Garcia, "Airtime usage control in virtualized multi-cell 802.11 networks," in IEEE Globecom Workshops (GC Wkshps). IEEE, Dec. 2015, pp. 1-6. 